\title{
Survivorship and clinical outcome of modular endoprosthetic reconstruction for neoplastic disease of the lower limb
}

\author{
E. R. Ahlmann, \\ L. R. Menendez, \\ C. Kermani, \\ H. Gotha
}

From the University of Southern California, Los Angeles, USA

\begin{abstract}
We reviewed retrospectively the results in 211 consecutive patients who had undergone limb salvage for bone neoplasia with endoprosthetic reconstruction of the proximal femur (96), distal femur (78), proximal tibia (30) and total femur (7). Their mean age was 50 years (11 to 86 ) and the mean follow-up period was 37.3 months (1 to 204). A total of 35 (16.6\%) prostheses failed. Overall, implant survival was $78 \%$ (95\% confidence interval (CI) 0.29 to $0.54)$ at five years, $60 \%(95 \% \mathrm{Cl} 0.93$ to 2.35$)$ at ten years and $60 \%(95 \% \mathrm{Cl} 1.27$ to 3.88$)$ at 15 years. Survivorship of the limb was $97.6 \%$ (95\% Cl 1.73 to 3.35$)$ at ten years. The gender, age, diagnosis and location of the tumour were not prognostic variables for failure. Modular endoprosthetic replacement in the lower limb is a durable long-term reconstructive option, with the implants generally outlasting the patient.
\end{abstract}

The distal femur is the most common site for primary bone tumours and the proximal femur is the most common location for metastatic lesions. ${ }^{1-2}$ Before the development of endoprostheses in the 1970s, the primary treatment for these lesions was amputation. With advances in radiation treatment and chemotherapy, limb salvage became an option in the early 1980s. Although there appears to be a higher incidence of local recurrence with limb salvage, the overall patient survival is similar to that for amputation. ${ }^{3}$ The development of new operative techniques, better patient selection and improved prosthetic design have been instrumental in improving the options for treatment.

The optimum method for reconstruction of the lower limb after resection of the femur or tibia is controversial. Options include the use of autografts, ${ }^{4-6}$ allografts, ${ }^{7,8}$ custom-made megaprostheses ${ }^{9-11}$ and modular endoprostheses. The first reconstructive procedures were performed using allograft or allograft-prosthetic composites..$^{12,13}$ Nonunion, fracture and infection often complicate the use of allografts. ${ }^{7,8}$ Custom-made endoprostheses were then developed which, while associated with fewer complications, were costly to manufacture. ${ }^{9-11}$ More recently modular endoprostheses have become available. These allow reconstruction of a wide variety of skeletal defects using off-the-shelf components without the expense or time required to manufacture a custom-made implant. Intra-operative assem- bly allows for the uncertainty of the margin of tumour resection, as well as offering an element of expandability when used for reconstruction in skeletally immature patients.

Improvements in the treatment of primary bone neoplasms have led to an increase in the long-term survival of the patients. Many of them are young and are expected to lead active lives, placing greater demands on their implants, whilst those with metastatic disease are anticipated to have poor bone quality, possibly placing a greater load on the endoprosthesis. Accordingly, durability of the implant is important in reducing the likelihood of revision.

We have studied the survivorship of modular endoprosthetic reconstructions in the treatment of both primary bone tumours and metastatic disease of the lower limb, and assessed the rate of revision, cause of failure and ultimate functional outcome.

\section{Patients and Methods}

Between July 1988 and November 2003, 235 patients with neoplasms of the lower limb were referred to our institution, of whom 24 underwent primary amputation. The results in the remaining 211 who had limb salvage with modular endoprosthetic reconstruction were reviewed retrospectively. The study was approved by the institutional review board. There were 102 men and 109 women with a mean age of 50 years (11 to 86). Of these, 89 had primary bone tumours, 86 metastatic dis- 
Table I. Diagnoses, current patient status and staging

\begin{tabular}{|c|c|c|c|c|c|c|}
\hline Diagnosis & Total patients Alive & Dead & Proximal femur & Distal femur & Proximal tibia & Total femur \\
\hline Metastatic disease & 86 & 74 & 72 & 10 & 0 & 4 \\
\hline Osteosarcoma & 35 & 7 & 6 & 29 & 6 & 1 \\
\hline Giant-cell tumour & 30 & 1 & 3 & 17 & 11 & 0 \\
\hline Chondrosarcoma & 14 & 6 & 5 & 6 & 3 & 0 \\
\hline Haemangiopericytoma & 7 & 2 & 2 & 4 & 1 & 0 \\
\hline Malignant fibrous histiocytoma & 5 & 1 & 0 & 3 & 3 & 0 \\
\hline Multiple myeloma & 1 & 5 & 4 & 1 & 0 & 1 \\
\hline Synovial sarcoma & 3 & 2 & 1 & 1 & 3 & 0 \\
\hline Ewing's sarcoma & 4 & 0 & 0 & 2 & 1 & 1 \\
\hline Lymphoma & 3 & 0 & 2 & 0 & 1 & 0 \\
\hline Enchondroma & 2 & 0 & 0 & 1 & 1 & 0 \\
\hline Fibrous dysplasia & 1 & 1 & 0 & 1 & 0 & 0 \\
\hline Liposarcoma & 0 & 1 & 1 & 0 & 0 & 0 \\
\hline Multiple osteochondromatosis & 1 & 0 & 0 & 1 & 0 & 0 \\
\hline Primitive neuro-ectodermal tumour & 1 & 0 & 0 & 1 & 0 & 0 \\
\hline Pigmented villonodular synovitis & 1 & 0 & 0 & 1 & 0 & 0 \\
\hline Total & 211 & 100 & 96 & 78 & 30 & 7 \\
\hline
\end{tabular}

ease and 36 had benign lesions. The diagnoses are shown in Table I.

At the time of the study 74 of the patients had died from metastases and 26 from their primary bone neoplasm. A minimum follow-up of one year was obtained in 173 patients, of two years in 114, of more than five years in 55 and of more than ten years in 22 patients. The mean length of follow-up for patients with metastatic disease was only 13.5 months (1 to 86.2 ) because of early death from the disease. By contrast, patients with primary bone tumours were followed for a mean of 59.5 months (1 to 204). The overall mean length of follow-up was 37.3 months (1 to 204).

All patients underwent staging which included plain radiography and MRI of the limb, CT of the chest and total body scintigraphy prior to biopsy. Patients with primary bone neoplasms were staged according to the system of Enneking, Spanier and Goodman, ${ }^{14}$ with 77 having stageIIB, eight stage-III and four stage-IB disease at presentation. There were 33 patients with benign bone tumours who had grade- 3 lesions and two who had grade-2 lesions. When indicated, patients received the standard pre-operative chemotherapy regime in use at the time of their treatment. Operative technique. All operations were carried out by a single surgeon (LRM). Tumour resection was performed with the goal of obtaining adequate disease-free margins. For resection of tumours of the proximal femur, biopsy tracts were excised en bloc with the specimen through a posterolateral approach to the hip. The abductor muscles were detached from the specimen by transection through the tendon or muscle. Wide resection with tumour-free margins was obtained in all patients. All resections of the hip were intra-articular. The Modular Segmental Replacement System (MSRS) endoprosthesis (Howmedica, Rutherford, New Jersey) was used in all patients to reconstruct the large segmental defects resulting from resection of the tumour. The articulation was bipolar in 62 patients and a total hip arthroplasty in 34 . This choice was based on pre- operative anticipated survival and intra-operative assessment of the acetabulum and surrounding soft-tissue attachments. All femoral stems were fixed with a polymethylmethacrylate (PMMA) cement mantle of $1 \mathrm{~mm}$ to $2 \mathrm{~mm}$, after reaming the host femur $2 \mathrm{~mm}$ larger than the diameter of the stem. Acetabular components, when used, were cemented. Reconstruction of the abductor muscles was performed by suturing the tendons to the remaining host soft tissue without direct attachment to the endoprosthesis.

For resection of tumours about the knee, a medial or lateral parapatellar arthrotomy was performed depending on the location of the lesion. For tumours arising from the proximal tibia, the insertion of the extensor mechanism on the tibial tuberosity was resected en bloc with the lesion. Wide tumour-free margins were obtained for all patients. A total of 105 resections of the knee were intra-articular and three were extra-articular. The MSRS endoprosthesis, with a rotating kinematic hinged knee mechanism, was used to reconstruct the segmental defects arising from resection of the tumour. A total of 78 patients had reconstruction for tumours of the distal femur and 30 for neoplasms of the proximal tibia. All the components were cemented to allow immediate post-operative weight-bearing. The stems were fixed with a PMMA cement mantle of $1 \mathrm{~mm}$ to $2 \mathrm{~mm}$ with the routine use of modern cementing techniques of pressurisation and an intramedullary cement plug. The patella was resurfaced routinely. Reconstruction of the extensor mechanism was performed after proximal tibial resection as described by Malawer and Sugarbaker. ${ }^{15}$ The advanced patellar tendon was directly sutured to the prosthesis using Dacron tape. A medial gastrocnemius transpositional muscle flap was then rotated anteriorly to cover the proximal tibia and knee, and was sutured to the underlying patellar tendon, quadriceps and joint capsule. The gastrocnemius flap ensured adequate soft-tissue coverage around the prosthesis and provided mechanical and biological reinforcement of the extensor mechanism. After distal femoral 
reconstruction a medial gastrocnemius rotational flap was transposed when adequate soft-tissue coverage could not be achieved locally.

Total femoral reconstruction was performed as described by Malawer and Sugarbaker. ${ }^{15}$ The hip was resected through a posterolateral approach, with transection of the abductors through their tendinous attachments. The incision was extended distally to the anterolateral aspect of the patellar tendon and tibial tuberosity. A transverse tibial osteotomy was performed approximately $1 \mathrm{~cm}$ below the joint line, as for a standard knee arthroplasty. Endoprosthetic reconstruction was performed with the MSRS system using bipolar hip components and a rotating kinematic hinge knee mechanism. The hip abductors were reconstructed by directly suturing the tendons to the remaining soft tissue. A medial gastrocnemius interposition flap was performed in all patients to provide adequate soft-tissue coverage about the knee.

Post-operatively, intravenous antibiotics were routinely administered until all drains were removed by the third post-operative day. Patients with proximal femoral and distal femoral reconstructions were allowed to bear full weight immediately after surgery without a brace or cast. Those who underwent distal femoral reconstruction began mobilisation of the knee on the second post-operative day. The protocol for patients who had undergone proximal tibial resection included immobilisation of the knee in full extension for three weeks to allow healing of the extensor mechanism. After removal of the knee brace, patients began physiotherapy, concentrating on knee extension exercises to prevent an extensor lag. Once full extension had been achieved, efforts were directed towards improving flexion of the knee. Patients who had total femoral replacement were mobilised post-operatively with an abduction brace and were restricted to partial weight-bearing. At four to six weeks, once active hip abduction had been achieved, the brace was discarded and full weight-bearing was allowed. The patients were reviewed every three months for the first two years after operation, then every six months for three additional years and annually thereafter.

Failure of the endoprosthetic reconstruction was defined as revision of any or all components of the implant, removal of the prosthesis or amputation of the limb. The records were inspected for the onset of any complications. Causes of failure leading to revision included infection, recurrence of the tumour, aseptic loosening, fatigue failure, peri-prosthetic fracture and dislocation.

The functional outcome was assessed in all surviving patients using the revised Musculoskeletal Tumour Society functional rating system. ${ }^{16}$ This uses a 30 -point scale to weight each of six parameters equally; these are pain, functional limitation, walking distance, use of support, emotional acceptance and gait. An investigator not involved in clinical care interviewed patients by telephone or at their latest follow-up and completed a questionnaire with each patient.

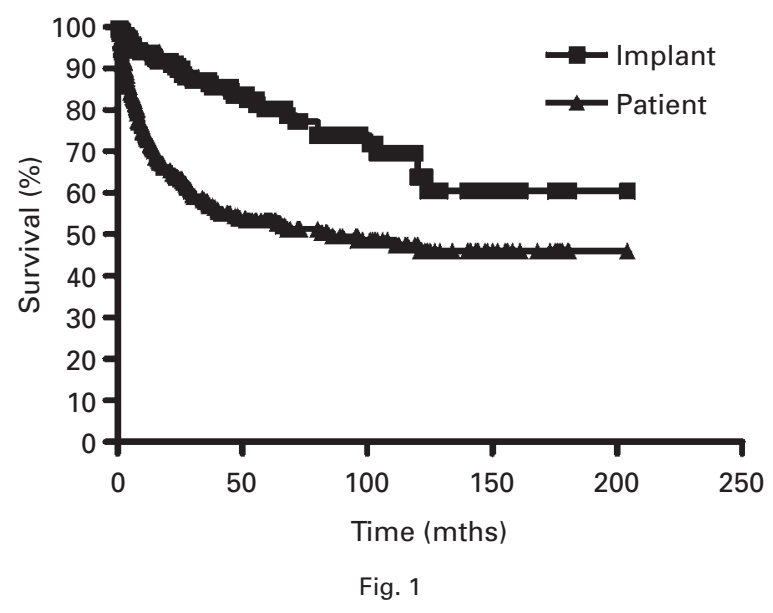

Kaplan-Meier survivorship curve for overall lower-limb survival of endoprostheses, compared with patient survival.

Statistical analysis. Kaplan-Meier survivorship curves with a $95 \%$ confidence interval (CI) for both implant and patient survival were prepared using the GraphPad Prism software (GraphPad Software Inc., San Diego, California). Patients who died with their original implant in place were censored. The starting point was defined as the date of implantation of the prosthesis and the end-point as the need for revision, removal of the implant or amputation. The end-point for limb survival was amputation. The log rank test and Cox proportional hazards regression model were used to analyse the association between the various clinical variables and failure of the implant. A p value of less than 0.05 was considered to be significant.

\section{Results}

A total of 35 prostheses $(16.6 \%)$ were deemed to be a failure at the time of the latest follow-up. The rate of revision was $13.8 \%$ (29), of amputation $2.4 \%$ (5) and of prosthetic removal $0.5 \%(1)$. The reasons for failure in these patients are outlined in Table II. Overall survivorship of the prosthesis based on Kaplan-Meier estimates was 78\% (95\% confidence interval (CI) 0.29 to 0.54 ) at five years, $60 \%$ $(95 \%$ CI 0.93 to 2.35$)$ at ten years, and $60 \%$ (95\% CI 1.27 to 3.88$)$ at 15 years. When compared with overall patient survival of $52 \%(95 \%$ CI 0.39 to 0.76$)$ at five years and $45 \%(95 \%$ CI 0.48 to 0.99$)$ at ten years, the endoprostheses greatly outlasted the patients in whom they had been implanted (Fig. 1). The survivorship for the proximal femoral replacements was $82 \%$ at both five (95\% CI 0.15 to 0.36 ) and ten (95\% CI 0.28 to 0.44 ) years. Survivorship for the distal femoral replacements was $75 \%(95 \%$ CI 0.57 to 2.01$)$ at five years and $58 \%$ (95\% CI 0.98 to 2.50 ) at ten years. For the proximal tibial replacement it was $82 \%(95 \%$ CI 0.77 to 2.35$)$ and $52 \%$ (95\% CI 0.98 to 4.04$)$ at five and ten years respectively. There were no failures in the total femoral replacements, 
Table II. Mode of failure based on location of the endoprosthesis

\begin{tabular}{lccccc}
\hline Mode of failure (n= 211) & Total patients (\%) & $\begin{array}{l}\text { Proximal femur (\%) } \\
\text { (n= 96) }\end{array}$ & $\begin{array}{l}\text { Distal femur (\%) } \\
\text { (n= 78) }\end{array}$ & $\begin{array}{l}\text { Proximal tibia (\%) } \\
\text { (n= 30) }\end{array}$ & Total femur \\
\hline Infection & $11(5.2)$ & $3(3.1)$ & $5(6.4)$ & $3(10)$ & 0 \\
Aseptic loosening & $5(2.4)$ & $0(0.0)$ & $4(5.1)$ & $1(3.3)$ & 0 \\
Peri-prosthetic fracture & $1(0.5)$ & $0(0.0)$ & $1(1.2)$ & $0(0.0)$ & 0 \\
Fatigue failure & $9(4.3)$ & $0(0.0)$ & $7(8.9)$ & $2(6.6)$ & 0 \\
Local recurrence & $6(2.8)$ & $3(3.1)$ & $0(0.0)$ & $0(0.0)$ & 0 \\
Instability/dislocation & $3(1.4)$ & $3(3.1)$ & $19(24.4)$ & $7(23.3)$ & 0 \\
Total & $35(16.6)$ & $9(9.3)$ & &
\end{tabular}

resulting in $100 \%$ survivorship at five and ten years. Gen$\operatorname{der}(\mathrm{p}=0.5811)$, age $(\mathrm{p}=0.8183)$, diagnosis $(\mathrm{p}=0.2372)$ and location of the tumour $(\mathrm{p}=0.6665)$ were not prognostic variables for failure. There was, however, a trend towards greater implant survivorship in females, older patients and in those with metastatic disease. The type of articulation of the femoral head in proximal femoral reconstruction was a significant prognostic indicator for failure of the implant $(\mathrm{p}=0.018)$, with bipolar implants having a greater survivorship than total hip endoprostheses. No difference was found in the rates of revision in those patients who died compared with those who survived $(p=0.9714)$. The mean age of the patients in whom the initial endoprosthetic reconstruction ultimately failed was 36 years ( 11 to 80$)$. The mean interval between the initial surgery and failure was 36 months (1 to 120 ).

There were five amputations $(2.4 \%)$, of which one was a hip disarticulation and four were above-knee. One patient with a proximal femoral endoprosthesis developed a deep infection and chose to have an amputation. Two further amputations, one after distal femoral and one after proximal tibial reconstruction, were performed for infection. The remaining two patients underwent above-knee amputations for local recurrence of tumour. One patient had a malignant fibrous histiocytoma of the proximal tibia, and the other an osteogenic sarcoma of the distal femur. The mean interval between endoprosthetic reconstruction and amputation was 23.4 months ( 3 to 48 ). Overall survivorship of the limb without amputation was $97.6 \%$ (95\% CI 1.73 to 3.35 ) at ten years.

There was local recurrence in six patients $(2.8 \%)$. In three this occurred at the proximal femur, in two at the distal femur and in one at the proximal tibia. Of those patients in whom there was recurrence at the proximal femur, one had a recurrent haemangioendothelioma and two developed recurrent metastases (renal-cell carcinoma, squamous-cell carcinoma). Two of these patients underwent revision, one to a cemented acetabulum and the other to a larger femoral component. The remaining patient had the implant removed but without re-implantation. Of the two patients with distal femoral recurrences, one underwent above-knee amputation and the other successful revision for recurrent osteogenic sarcoma. The single patient with a proximal tibial recurrence had an amputation. The mean time from initial resection to recurrence was 21 months (2.3 to 36.5 ). At the time of this study all patients with local recurrence had died from their disease.

Another leading cause of failure resulting in revision was instability. Ten dislocations of proximal femoral endoprostheses occurred with three resulting in revision of the implant. The remaining patients were successfully treated by either closed (two patients) or open reduction (five patients). Of the dislocations which occurred, four were in bipolar replacements and six were in total hip arthroplasties (THA). Patients with THA were more prone to instability (six of 34 patients; $17.6 \%$ ) than those with bipolar replacements (four of 62 patients; $6.5 \%$ ). In six patients dislocation occurred within two months of the initial procedure as a result of a fall. Overall, the mean time from endoprosthetic replacement to dislocation was 4.7 months (1 to 15.2). Instability was not found after any distal femoral, proximal tibial or total femoral reconstructions.

Prosthetic infection was the cause of failure in 11 patients $(5.2 \%)$. Three underwent amputation as definitive management while eight were successfully treated by twostage revision. Most infections developed relatively soon after the initial endoprosthetic reconstruction after a mean interval of six months (2.5 to 55.6).

There was fatigue failure of the implant in nine patients $(4.3 \%)$, in seven of whom it occurred after distal femoral reconstruction and in two after proximal tibial. Breakage of the axle of the rotating kinematic knee hinge accounted for four failures. The remaining five were all fatigue fractures of the femoral stem. All patients were successfully revised at a mean of 50.6 months (2.3 to 120$)$ from the initial reconstruction.

Aseptic loosening was an infrequent cause of failure with only five occurrences $(2.4 \%)$. Four distal femoral stems and one proximal tibial stem were successfully revised at a mean 80.4 months (38 to 120 ).

Peri-prosthetic fracture occurred in a 17 -year-old man who was involved in a motor vehicle accident and sustained a femoral fracture just proximal to the stem of a distal femoral endoprosthesis. The implant was revised to a longer stem 46.7 months after the initial reconstruction.

The mean MSTS functional outcome scores ${ }^{16}$ for those patients surviving at the time of this study was 22.25 (14 to 30 ), indicating that they were able to achieve $74 \%$ of nor- 
mal function. There was no significant difference $(\mathrm{p}=$ 0.4438) in MSTS scores based on the location of the reconstruction with a mean functional score for the proximal femoral replacement of $22(73.3 \%)$, distal femoral replacements of $22.9(76.2 \%)$, proximal tibial replacements of $22.25(74 \%)$, and total femoral replacements of 19.5 (65\%).

\section{Discussion}

We have used modular segmental reconstruction after resection of neoplastic disease in the lower limb since 1988. Modularity allows intra-operative adjustment when greater resection is necessary than previously anticipated, offers the possibility of expanding the implants in skeletally immature patients to allow for growth, and decreases the cost in comparison with that of custom-made implants. This series describes the results of a single type of modular endoprosthesis. Previous studies have included results of a variety of modular and custom-made implants or have been confined to custom-made implants alone.

The rate of failure in the current series was $16.6 \%$, with five-, ten-, and 15 -year survivorship of the implant of $78 \%$ (95\% CI 0.29 to 0.54 ), $60 \%$ (95\% CI 0.93 to 2.35 ), and $60 \%$ (95\% CI 1.27 to 3.88 ), respectively, which compares favourably with those of custom-made implants. Dobbs et $\mathrm{al}^{17}$ reported the results in 81 patients treated by a custommade endoprosthetic replacement of the proximal femur. The five- and ten-year survivorship of the implant was reported as $73 \%$ and $63 \%$, respectively. Horowitz et al ${ }^{18}$ described the results of 93 patients who underwent implantation of either a custom-made or standard lowerlimb endoprosthesis. Survivorship of the implant at five years was $88 \%$ for the proximal femoral replacement, $59 \%$ for the distal femoral prosthesis and 54\% for the proximal tibial implant. In a study by Unwin et $\mathrm{al}^{11}$ in which 232 patients had custom-made proximal femoral replacements, the survivorship was $68 \%$ at ten years. In the same study, the results of 493 distal femoral replacements with a custom-made implant based on a fully constrained knee showed a probability of $67 \%$ of surviving aseptic loosening at ten years. Roberts et $\mathrm{a}^{19}$ found a distal femoral survivorship of $64 \%$ at seven years and Rougraff et al ${ }^{20}$ of $66 \%$ at ten years. Malawer and $\mathrm{Chou}^{21}$ noted that their proximal tibial reconstructions fared the worst with a crude five- and ten-year survivorship of $45 \%$. Similarly, Torbert et al ${ }^{22}$ observed that reconstructions of the proximal tibia with survival rates of $63 \%$ at both five and ten years, were more likely to fail than reconstructions of the proximal or distal femur. There are few survivorship studies of total femoral replacements, but $100 \%$ survivorship has been reported at approximately two, ${ }^{15}$ four ${ }^{23}$ and ten years. ${ }^{24}$ Our five- and ten-year survivorship, respectively, of $82 \%$ at the proximal femur, $75 \%$ and $58 \%$ at the distal femur, $82 \%$ and $52 \%$ at the proximal tibia and $100 \%$ for total femoral reconstruction, indicates that the modular system used in our study compares favourably with a wide variety of other implants.
Although the proximal femur is one of the most successful sites of endoprosthetic reconstruction, instability remains the most common complication associated with large segmental reconstruction of the proximal femur and is a frequent cause of failure. Several factors influence the rate of dislocation including acetabular resurfacing, resection of the joint capsule and the technique of abductor repair. A slightly higher rate of dislocation has been reported after THA than after bipolar replacement. Rates of dislocation of $11 \%$ to $15 \%$ have been described when the acetabulum has been resurfaced..$^{9,25}$ In our series, dislocation was almost three times more common after THA than after bipolar replacement. Bickels et $\mathrm{al}^{26}$ noted the results in 57 patients who underwent bipolar or unipolar reconstruction of the proximal femur with acetabular preservation, capsular repair and anatomical reconstruction of the abductor mechanism to the endoprosthesis. Only one patient developed a dislocation. An additional contributing factor predisposing to instability is abductor weakness. Tumour resection about the hip often requires that the abductor muscles be sacrificed, meaning that reconstruction to the endoprosthesis is not possible. We did not reattach the abductors directly to the prosthesis, but instead sutured them to the remaining soft tissues. The literature suggests that the most effective method of restoring stability of the hip is acetabular preservation, capsulorrhaphy and abductor reconstruction, directly to the endoprosthesis when feasible.

Infection occurred in $5.2 \%$ of patients in our study, which is similar to other series with rates ranging from $3 \%$ to $13 \%,{ }^{10,27,28}$ but better than allograft reconstruction in which rates as high as $30 \%$ have been reported. ${ }^{8}$ Malawer and $\mathrm{Chou}^{21}$ proposed administering long-term suppressive antibiotics during chemotherapy and other periods of immunosuppression to decrease the rate of infection. Another important factor in reducing the rate of infection is the use of rotational or free muscle flaps to provide adequate soft-tissue coverage around the endoprosthetic reconstructions.

The local rate of recurrence in our series of $2.8 \%$ compares favourably with that seen in other studies. Previous reports noted rates of recurrence ranging from 3\% to $25 \% .{ }^{22,28-30}$ Risk factors associated with increased recurrence have been shown to include the margin of resection, pathological fracture, poor response to chemotherapy and intravascular extension of the tumour. ${ }^{31}$

In our study the rate of aseptic loosening was $2.4 \%$ (five patients). Mechanical failure occurred in nine patients $(4.3 \%)$ and one $(0.5 \%)$ had a peri-prosthetic fracture. The rates of mechanical failure have been noted previously to range from $5 \%$ to $16 \% 27,28,32,33$ and of aseptic loosening from $7 \%$ to $27 \% .^{10,11,26,34}$ Frink et $\mathrm{al}^{33}$ recently published a follow-up for more than five years of 74 patients treated by a kinematic rotating/hinge distal femoral endoprosthesis in which there was one failure of the patellar component, one failure of an all-polyethylene tibial component, one 
fracture of the femoral stem and one axle breakage. Our mechanical failures occurred early in the use of this system, with most before 1995. Improved durability may be related to advances in cementing techniques, such as pressurisation and distal plugging used throughout in our patients, as well as improvements in prosthetic design and metallurgy. The decreased incidence of fatigue failure of the stem may be related to several changes of design, including forging rather than casting to increase in strength and the use of stronger alloys. ${ }^{35}$ Surfaces which encourage extramedullary porous ingrowth on more recently developed implants allow for soft-tissue ongrowth and the option for extracortical bone graft. This may reduce the rate of aseptic loosening by allowing load sharing with the prosthesis.

Modular endoprosthetic replacement of the lower limb is a durable and long-term reconstructive option after resection of a tumour. For most patients with neoplastic disease these implants will generally outlast the patient and provide acceptable function and quality of life.

No benefits in any form have been received or will be received from a commercial party related directly or indirectly to the subject of this article.

\section{References}

1. Harrington KD. Orthopaedic management of metastatic bone disease. St. Louis: Mosby, 1988:143-7.

2. Schneiderbauer MM, von Knoch M, Schleck CD, et al. Patient survival after hip arthroplasty for metastatic disease of the hip. J Bone Joint Surg [Am] 2004;86-A 1684-9.

3. Simon MA, Aschliman MA, Thomas N, Mankin HJ. Limb-salvage treatment versus amputation for osteosarcoma of the distal end of the femur. J Bone Joint Surg [Am] 1986;68-A:1331-7.

4. Chen WM, Chen TH, Huang CK, Chiang CC, Lo WH. Treatment of malignant bone tumours by extracorporeally irradiated autograft-prosthetic composite arthroplasty. J Bone Joint Surg [Br] 2002;84-B:1156-61.

5. Smith WS, StruhI S. Replantation of an autoclaved autogenous segment of bone for treatment of chondrosarcoma: long-term follow-up. J Bone Joint Surg [Am] 1988; 70-A:70-5.

6. Harrington KD, Johnston JO, Kaufer HN, Luck JV Jr, Moore TM. Limb salvage and prosthetic joint reconstruction for low-grade and selected high-grade sarcomas of bone after wide resection and replacement by autoclaved (corrected) autogeneic grafts. Clin Orthop 1986;211:180-214.

7. Fox EJ, Hau MA, Gebhardt MC, et al. Long-term followup of proximal femoral allografts. Clin Orthop 2002;397:106-13.

8. Gebhardt MC, Flugstad DI, Springfield DS, Mankin HJ. The use of bone allografts for limb salvage in high-grade extremity osteosarcoma. Clin Orthop 1991 270:181-96.

9. Kabukcuoglu Y, Grimer RJ, Tillman RM, Carter SR. Endoprosthetic replacement for primary malignant tumors of the proximal femur. Clin Orthop 1999;358:8-14.

10. Mittermayer F, Krepler P, Diminkus $\mathbf{M}$, et al. Long-term followup of uncemented tumor endoprostheses for the lower extremity. Clin Orthop 2001;388:167-77.

11. Unwin PS, Cannon SR, Grimer RJ, et al. Aseptic loosening in cemented custom made prosthetic replacements for bone tumors of the lower limb. J Bone Joint Surg [Br] 1996;78-B:5-13
12. Donati D, Giacomini S, Gozzi E, Mercuri M. Proximal femur reconstruction by an allograft prosthesis composite. Clin Orthop 2002;394:192-200.

13. Gitelis S, Piasecki P. Allograft prosthetic composite arthroplasty for osteosarcoma and other aggressive bone tumors. Clin Orthop 1991;270:197-201.

14. Enneking WF, Spanier SS, Goodman MA. A system for the surgical staging of musculoskeletal sarcoma. Clin Orthop 1980;153:106-20.

15. Malawer MM, Sugarbaker PH. Musculoskeletal cancer surgery: treatment of sar comas and allied diseases. Boston: Kluwer Academic Publishers, 2001:483-502.

16. Enneking WF, Dunham W, Gebhardt MM, Malawer MM, Pritchard DJ. A system for functional evaluation for reconstructive procedures after surgical treatment of tumours of the musculoskeletal system. Clin Orthop 1993;286:241-6.

17. Dobbs HS, Scales JT, Wilson JN, et al. Endoprosthetic replacement of the proximal femur and acetabulum. J Bone Joint Surg [Br] 1981;63-B:219-24

18. Horowitz SM, Glasser DB, Lane JM, Healey JH. Prosthetic and extremity survivorship after limb salvage for sarcoma: how long do the reconstructions last? Clin Orthop 1993;293:280-6.

19. Roberts P, Chan D, Grimer RJ, Sneath RS, Scales JT. Prosthetic replacement of the distal femur for primary bone tumors. J Bone Joint Surg [Br] 1991;73-B:762-70.

20. Rougraff BT, Simon MA, KneisI JS, Greenberg DB, Mankin HJ. Limb salvage compared with amputation for osteosarcoma of the distal end of the femur: a long term oncological, functional, and quality of life study. J Bone Joint Surg [Am] 1994 76-A:649-61.

21. Malawer MM, Chou LB. Prosthetic survival and clinical results with use of largesegment replacements in the treatment of high-grade bone sarcomas. J Bone Joint Surg [Am] 1995;77-A:1154-66.

22. Torbert JT, Fox EJ, Hosalkar HS, Ogilvie CM, Lackman RD. Endoprosthetic reconstructions: results of long-term followup of 139 patients. Clin Orthop 2005;438: $51-9$

23. Mankin HJ, Hornicek FJ, Harris $\mathbf{M}$. Total femoral replacement procedures in tumor treatment. Clin Orthop 2005;438:60-4.

24. Nakamura S, Kusuzaki K, Murata H, et al. More than 10 years of follow-up of two patients after total femur replacement for malignant bone tumor. Int Orthop 2000;24:176-8.

25. Rechl H, Reinisch M, Plotz W, Burgkart R, Gradinger R. Soft tissue reconstruction about the proximal femur. Oper Tecn Orthop 1999:9:115-19.

26. Bickels J, Meller I, Henshaw RM, Malawer MM. Reconstruction of hip stability after proximal and total femur resections. Clin Orthop 2000;375:218-30.

27. Eckardt JJ, Eilbert FR, Rosen G, et al. Endoprosthetic replacement for stage IIB osteosarcoma. Clin Orthop 1991;70:202-13.

28. Quill G, Gitelis S, Morton T, Piasecki P. Complications associated with limb salvage for extremity sarcomas and their management. Clin Orthop 1990;260:242-50

29. Guilano AE, Eilbert FR, Morton DL. The management of locally recurrent softtissue sarcoma. Ann Surg 1982;196:87-91.

30. Karakousis CP, Emrich LJ, Rao U, Krishnamsetty RM. Feasibility of limb salvage and survival in soft tissue sarcomas. Cancer 1986:57:484-91.

31. Ward WG, Eckardt JJ, Dorey F, et al. Local recurrence following surgical treatment of 242 primary malignant bone tumors: an analysis of 39 cases. Orthop Trans 1994;18:27

32. Wirganowicz PZ, Eckardt JJ, Dorey FJ, Eilbert FR, Kabo JM. Etiology and results of tumor endoprosthesis revision surgery in 64 patients. Clin Orthop 1999;358: 64-74.

33. Frink SJ, Rutledge J, Lewis Vo, Lin PP, Yasko AW. Favourable long-term results of prosthetic arthroplasty of the knee for distal femur neoplasms. Clin Orthop 2005 438:65-70.

34. Kawai A, Lin PP, Boland PJ, Athanasian EA, Healey JH. Relationship between magnitude of resection, complication, and prosthetic survival after prosthetic knee reconstructions for distal femoral tumors. J Surg Orthop 1999;70:109-15.

35. Plotz W, Rechl H, Burgkart R, et al. Limb salvage with tumor endoprostheses for malignant tumors of the knee. Clin Orthop 2002;405:207-15 